Check for updates

Cite this: J. Mater. Chem. C, 2018, 6, 8546

Received 22nd May 2018, Accepted 23rd June 2018 DOI: $10.1039 / c 8 t c 02481 b$

rsc.li/materials-c

\section{Enhanced thermoelectric performance of Sn-doped $\mathrm{Cu}_{3} \mathrm{SbS}_{4} \dagger$}

\author{
Kan Chen, (D) *a Cono Di Paola, ${ }^{b}$ Baoli Du, ${ }^{c}$ Ruizhi Zhang, ${ }^{a}$ Savio Laricchia, ${ }^{b}$ \\ Nicola Bonini, ${ }^{b}$ Cedric Weber, ${ }^{b}$ Isaac Abrahams, (D) ${ }^{d}$ Haixue Yan (D) and \\ Mike Reece ${ }^{a}$
}

$\mathrm{Cu}_{3} \mathrm{SbS}_{4}$ is an earth-abundant and low-cost alternative thermoelectric material for medium temperature applications. Tin doping into $\mathrm{Cu}_{3} \mathrm{SbS}_{4}$ yields materials with high thermoelectric performance. The electronic structure of $\mathrm{Sn}$-doped $\mathrm{Cu}_{3} \mathrm{SbS}_{4}$ was studied using both hybrid density functional theory (DFT) and the quasi-particle self-consistent GW (QSGW) approach. A synthesis method involving mechanical alloying (MA) and spark plasma sintering (SPS) was employed to produce dense and single phase $\mathrm{Cu}_{3} \mathrm{SbS}_{4}$ samples with very fine grain size. Previously unreported nano-scale twins on $\{112\}$ planes were observed by transmission electron microscopy (TEM). All of the samples showed very low lattice thermal conductivity, which is attributed to their microstructures. Sn was found to substitute Sb successfully in $\mathrm{Cu}_{3} \mathrm{SbS}_{4}$ and work effectively as an acceptor dopant, leading to an enhanced power factor. A maximum $z T$ value of 0.72 at $623 \mathrm{~K}$ was achieved in $\mathrm{Cu}_{3} \mathrm{Sb}_{1-x} \mathrm{Sn}_{x} \mathrm{~S}_{4}(x=0.05)$, which is comparable to the Se analogue $\mathrm{Cu}_{3} \mathrm{SbSe}_{4}$.

\section{Introduction}

Global energy production is subject to considerable losses every year, mainly in the form of waste heat. ${ }^{1}$ Finding an effective way to harvest waste heat is of great importance to mitigate the rising issues related to energy resources and the environment. Thermoelectric generators (TEGs) based on the Seebeck effect can directly convert waste heat into electricity from a wide variety of heat sources. ${ }^{2}$ Unlike traditional heat pumps, TEGs are solid-state devices which are reliable, compact and emission-free. Currently, their applications are very restricted due to their relatively low conversion efficiency and high-cost. The conversion efficiency of a TEG is primarily determined by the dimensionless figure-of-merit, $z T=\alpha^{2} \rho^{-1} \kappa^{-1} T$, of the constituent thermoelectric (TE) material, where $T$ is the absolute temperature, $\alpha$ is the Seebeck coefficient, $\rho$ is the electrical resistivity, and $\kappa$ is the thermal conductivity. ${ }^{2-5}$ In order to make a cost-effective TEG for the mass market, continuous efforts are being made to push up the $z T$ value of known systems, but most of the good thermoelectric materials

\footnotetext{
${ }^{a}$ School of Engineering and Materials Science, Queen Mary University of London, Mile End Road, London E1 4NS, UK. E-mail: kan.chen@qmul.ac.uk

${ }^{b}$ Department of Physics, King's College London, London WC2R 2LS, UK

${ }^{c}$ School of Physics and Electronic Information Engineering,

Henan Polytechnic University, Jiaozuo 454000, China

${ }^{d}$ School of Biological and Chemical Sciences, Queen Mary University of London, Mile End Road, London E1 4NS, UK

$\dagger$ Electronic supplementary information (ESI) available. See DOI: 10.1039/ c8tc02481b
}

contain toxic, expensive and rare elements. Thus, exploring new TE materials from the viewpoints of low-cost, low-toxicity and earth-abundance is drawing increasing attention. In recent years, Cu-based sulfides, such as $\mathrm{Cu}_{12} \mathrm{Sb}_{4} \mathrm{~S}_{13},{ }^{6-8} \mathrm{Cu}_{3} \mathrm{SbS}_{4},{ }^{9-11}$ $\mathrm{CuSbS}_{2},{ }^{12,13} \mathrm{CuCr}_{2} \mathrm{~S}_{4},{ }^{14} \mathrm{Cu}_{2} \mathrm{SnS}_{3},{ }^{15,16} \mathrm{Cu}_{3} \mathrm{SnS}_{4},{ }^{17} \mathrm{CuFeS}_{2},{ }^{18-21}$ $\mathrm{Cu}_{5} \mathrm{FeS}_{4},{ }^{22} \mathrm{Cu}_{6} \mathrm{Fe}_{2} \mathrm{SnS}_{8},{ }^{23}$ have been recognized as interesting and promising TE materials. Among these compounds, $\mathrm{Cu}_{3} \mathrm{SbS}_{4}$ has a simple zinc-blende related structure with a moderate band gap of $\sim 0.47 \mathrm{eV} .^{24}$ It shows a large effective mass derived from its degenerate bands, which is favourable for high thermoelectric performance. ${ }^{25}$ In our previous work, a combined theoretical and experimental study of $\mathrm{Cu}_{3} \mathrm{SbS}_{4}$ revealed its low thermal conductivity and promising TE performance. ${ }^{9}$ The $z T$ value of $\mathrm{Cu}_{3} \mathrm{SbS}_{4}$ was enhanced by Ge-doping on the $\mathrm{Sb}$ site, however, a competitive second phase of $\mathrm{Cu}_{2} \mathrm{GeS}_{3}$ was formed even at a very low dopant concentration $(\sim 1.25 \%)$. To further explore the potential TE properties of $\mathrm{Cu}_{3} \mathrm{SbS}_{4}$, we report here a detailed study of $\mathrm{Sn}$-doped $\mathrm{Cu}_{3} \mathrm{SbS}_{4}$. Although $\mathrm{Sn}$ has been previously reported as dopant in $\mathrm{Cu}_{3} \mathrm{SbS}_{4},{ }^{11}$ a complex solid-reaction method was used and the samples contained $\mathrm{SnO}_{2}$ impurity and large voids $(1 \mu \mathrm{m})$. A poor maximum $z T$ value of 0.1 was obtained at $573 \mathrm{~K}$. Based on our previous work on $\mathrm{Cu}_{3} \mathrm{SbS}_{4}$ and the reports on its analogue $\mathrm{Cu}_{3} \mathrm{SbSe}_{4},{ }^{26,27}$ a better TE performance is expected for Sn-doped $\mathrm{Cu}_{3} \mathrm{SbS}_{4} . \mathrm{Sn}^{4+}$ and $\mathrm{Sb}^{5+}$ have similar ionic radii and $\mathrm{Sn}$ has one less valence electron than $\mathrm{Sb}$, which suggests that Sn should be a good acceptor dopant in $\mathrm{Cu}_{3} \mathrm{SbS}_{4}$. In this work, we demonstrate high TE performance in Sn-doped $\mathrm{Cu}_{3} \mathrm{SbS}_{4}$ prepared by a combination of mechanical alloying (MA) and 
spark plasma sintering (SPS). This simple method produced samples of high-quality, high-purity and fine microstructures. $\mathrm{Sn}$ successfully substituted $\mathrm{Sb}$ and worked as a very effective p-type dopant in $\mathrm{Cu}_{3} \mathrm{SbS}_{4}$. This is further confirmed by detailed electronic structure calculations performed with hybrid DFT and validated with Quasi-particle Self-consistent GW (QSGW) calculations. Microstructural analysis reveals nano-twinning defects in $\mathrm{Cu}_{3} \mathrm{SbS}_{4}$, which have not previously been reported.

\section{Experimental}

\subsection{Synthesis and characterization}

Polycrystalline $\mathrm{Cu}_{3} \mathrm{Sb}_{1-x} \mathrm{Sn}_{x} \mathrm{~S}_{4}$ samples with nominal compositions corresponding to $x=0.00,0.01,0.03,0.05,0.10$ and 0.15 were prepared by MA + SPS. Starting powders of $\mathrm{Cu}$ (99.5\%, AlfaAesar), $\mathrm{Sb}$ (99.5\%, AlfaAesar), Sn (99.999\%, AlfaAesar), and S (reagent grade, purified by sublimation, Sigma-Aldrich) were loaded into a stainless steel jar with stainless steel balls and sealed in an inert atmosphere of argon. The powder mixture was milled at a rotation speed of $420 \mathrm{rpm}$ for $20 \mathrm{~h}$ in a planetary ball mill machine (QM-3SP2, Nanjing University, China). The as-synthesized powders were sintered at $723 \mathrm{~K}$ for $3 \mathrm{~min}$ at a pressure of $50 \mathrm{MPa}$ using an SPS furnace (FCT HPD 25, FCT System GmbH, Germany). The phases of the samples were examined using X-ray powder diffraction (XRD, PANalytical X'Pert Pro, $\mathrm{Cu}-\mathrm{K} \alpha$ ). The microstructures were observed using scanning electron microscopy (SEM, FEI, Inspect F, $10 \mathrm{kV}$ ) and transmission electron microscopy (TEM, Jeol JEM 2010, $200 \mathrm{kV}$ ). SEM images were taken of freshly fractured surfaces and TEM images were taken of the crushed powders. Bars of $3 \mathrm{~mm} \times 3 \mathrm{~mm} \times 15 \mathrm{~mm}$ were cut from the samples using a diamond saw for electrical resistivity $(\rho)$ and Seebeck coefficient $(\alpha)$ measurements, which were performed using a ZEM-3 (ULVAC-RIKO, Japan) from ambient temperature to $623 \mathrm{~K}$. The uncertainty of resistivity and Seebeck coefficient values is less than $5 \%$. The room temperature Hall coefficient $\left(R_{\mathrm{H}}\right)$ was measured using the Van der Pauw method (The Lake Shore 8400 Series HMS). The carrier concentration $(n)$ was calculated as $n=1 / e R_{\mathrm{H}}$. The uncertainty of the Hall measurement increased with increasing conductivity of the sample and the largest uncertainty was estimated to be $10 \%$. Thermal diffusivity was measured using the flash diffusivity method (LFA 457, Netzsch) up to $623 \mathrm{~K}$. The specific heat capacity $\left(C_{\mathrm{p}}\right)$ was determined by a comparative method in the LFA 457, using Pyroceram 9606 as a reference sample. The uncertainty of thermal diffusivity and heat capacity measurements was less than $5 \%$. The density was measured using the Archimedes method with an uncertainty of less than $1 \%$ and all the samples had a relative density greater than $98 \%$. The thermal conductivity was calculated using the thermal diffusivity, specific heat capacity and density.

\subsection{Computational methods}

The theoretical calculations were performed within the density functional theory (DFT) using the projector augmented wave method (PAW) as implemented in the Vienna ab initio simulation package (VASP). ${ }^{28,29}$ The screened hybrid functional of
Heyd, Scuseria and Ernzerhof (HSE06) and spin-orbit effects were also included in the calculations. ${ }^{30-33}$ A primitive cell of 8 atoms was used to describe the $\mathrm{Cu}_{3} \mathrm{SbS}_{4}$ crystal structure, while a supercell of 64 atoms $(2 \times 2 \times 2)$ was built to model Sn-doped $\mathrm{Cu}_{3} \mathrm{Sb}_{1-x} \mathrm{Sn}_{x} \mathrm{~S}_{4}(x=0.125)$, with a Sn atom substituted for an $\mathrm{Sb}$ atom. The Seebeck coefficient was computed within the constant relaxation time approximation using the Wannier interpolation scheme implemented in the BoltzWann code. ${ }^{34}$ Doping was simulated via a shift of the chemical potential. In order to benchmark the electronic structures obtained with HSE06, the band structure of $\mathrm{Cu}_{3} \mathrm{SbS}_{4}$ was also calculated with quasi-particle self-consistent $\mathrm{GW}$ (QSGW) as implemented in the Questaal package (The QUESTAAL code is freely available at http://www.questaal.org.), within a Full Potential Linear Muffin-Tin Orbital (FP-LMTO) framework. ${ }^{35-37}$ The smoothed LMTO basis used in this work includes atomic orbitals with $l \leq l_{\max }=4$, with $4 \mathrm{~d}$ orbitals of $\mathrm{Sb}$ and $\mathrm{Cu}$ added in the form of local orbitals. Since QSGW gives the self-consistent solution at the scalar relativistic level, we account for the spin-orbit coupling adding the operator $H_{\mathrm{SO}}=L \cdot S / 2 c^{2}$ as a perturbation not included in the self-consistency cycle. The QSGW calculations were performed using the HSE06 relaxed structure of $\mathrm{Cu}_{3} \mathrm{SbS}_{4}$.

\section{Results and discussion}

The X-ray powder diffraction patterns for the compositions studied in the $\mathrm{Cu}_{3} \mathrm{Sb}_{1-x} \mathrm{Sn}_{x} \mathrm{~S}_{4}$ system are shown in Fig. 1a. All of the compositions are phase pure (within the limits of the technique), exhibiting the tetragonal structure of $\mathrm{Cu}_{3} \mathrm{SbS}_{4}(\mathrm{PDF}$ 00-035-0581). The unit cell volume generally decreased (Fig. 1b) with increasing $x$-value although the volume change is very small (less than $0.4 \%$ ). According to Shannon, ${ }^{38}$ the radius for tetrahedral $\mathrm{Sn}^{4+}$ in a chalcogenide is $0.55 \AA$. Unfortunately, the corresponding value for $\mathrm{Sb}^{5+}$ is not recorded. However, using the reported Sb-S interatomic distance $(2.405 \AA)$ in $\mathrm{Cu}_{3} \mathrm{SbS}_{4}{ }^{39}$ and the Shannon ionic radius of $\mathrm{S}^{2-}(1.84 \AA)$, the radius of $\mathrm{Sb}^{5+}$ in a tetrahedral coordination is calculated to be $0.565 \AA$.
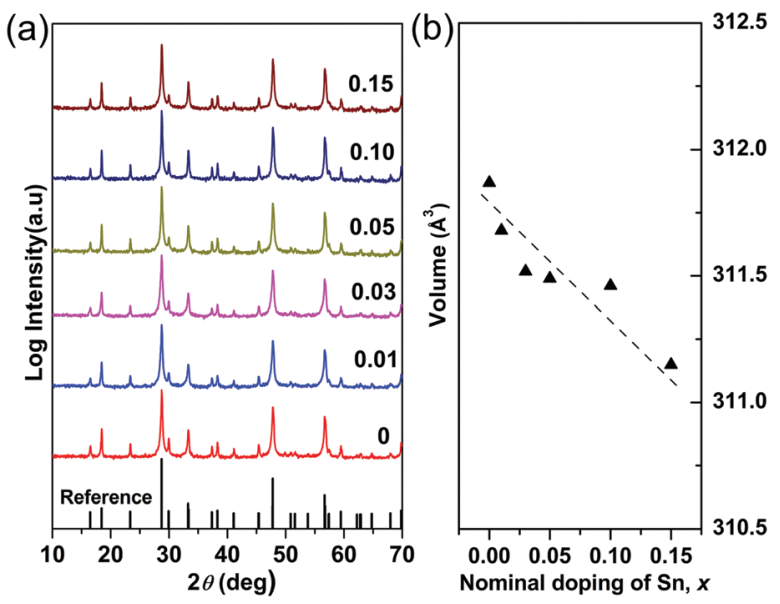

Fig. 1 (a) XRD patterns and (b) compositional variation of unit cell volume in $\mathrm{Cu}_{3} \mathrm{Sb}_{1-x} \mathrm{Sn}_{x} \mathrm{~S}_{4}$ system. 
Thus the decrease in unit cell volume appears to be consistent with substitution of $\mathrm{Sb}^{5+}$ by $\mathrm{Sn}^{4+}$ on the tetrahedral sites.

To gain further insight into the solubility as well as the structural effects related to Sn-doping, a theoretical analysis was performed using DFT. The lattice parameters of tetragonal $\mathrm{Cu}_{3} \mathrm{SbS}_{4}$ obtained with DFT are $a=5.41 \AA$ and $c=10.80 \AA$, which are in close agreement with the experimental data $(a=5.39 \AA$, $c=10.75 \AA)$. A model of $\mathrm{Cu}_{3} \mathrm{Sb}_{0.875} \mathrm{Sn}_{0.125} \mathrm{~S}_{4}(x=0.125)$ was built to illustrate the effects of Sn-doping. The calculated volume of $\mathrm{Cu}_{3} \mathrm{Sb}_{0.875} \mathrm{Sn}_{0.125} \mathrm{~S}_{4}$ was contracted by $0.4 \%$ with respect to the undoped $\mathrm{Cu}_{3} \mathrm{SbS}_{4}$, which is consistent with the experimental results. The calculated formation energy of the $\mathrm{Sn}_{\mathrm{Sb}}$ defect is $-0.61 \mathrm{eV}$, showing that solubility of $\mathrm{Sn}$ in $\mathrm{Cu}_{3} \mathrm{SbS}_{4}$ is energetically favourable. The formation energy obtained here is quite close to the formation energy of $\mathrm{Ge}_{\mathrm{Sb}}$ defects in $\mathrm{Cu}_{3} \mathrm{SbS}_{4}(-0.68 \mathrm{eV}){ }^{9}$ However, it is important to point out that, contrary to the case of Ge substitution, here the local restructuring around the Sn atom is very small (the $\mathrm{Sn}-\mathrm{Cu}$ distances contract by about $0.4 \%$ and the $\mathrm{Sn}-\mathrm{S}$ distances expand by about $0.5 \%$ upon relaxation from the pristine $\mathrm{Cu}_{3} \mathrm{SbS}_{4}$ geometry), which is in line with the good solubility observed experimentally.

$\mathrm{As} \mathrm{Sb}^{5+}$ is substituted by $\mathrm{Sn}^{4+}$, each $\mathrm{Sn}$ is expected to donate one hole in $\mathrm{Cu}_{3} \mathrm{SbS}_{4}$ by electronic compensation. Based on this assumption, the theoretical carrier concentration was calculated according to the nominal doping content, and is shown as the dashed line in Fig. 2. The measured carrier concentrations are shown as red circles, showing good consistency between the theoretical and the experimental results. This further suggests that $\mathrm{Sn}$ completely substitutes for $\mathrm{Sb}$ in $\mathrm{Cu}_{3} \mathrm{SbS}_{4}$. In fact, a complete solid-solution of $\mathrm{Cu}_{3} \mathrm{SbS}_{4}-\mathrm{Cu}_{3} \mathrm{SnS}_{4}$ was successfully prepared. The samples with high $\mathrm{Sn}$-content are very conductive and exhibit poor thermoelectric performance and therefore not included in this work. However, the mechanisms and effects of this high solubility might provide a more insightful understanding of $\mathrm{Cu}_{3} \mathrm{SbS}_{4}$ and related compounds such as $\mathrm{Cu}_{2} \mathrm{ZnSnS}_{4}$ (Kesterite), and this part is of a parallel investigation. ${ }^{40}$

Fig. 3 shows typical fracture surfaces of the undoped and Sn-doped samples. Both transgranular and intergranular fractures are observed. The samples have very fine grains and the grain size estimated from SEM of fracture surfaces is about $50 \mathrm{~nm}$ to

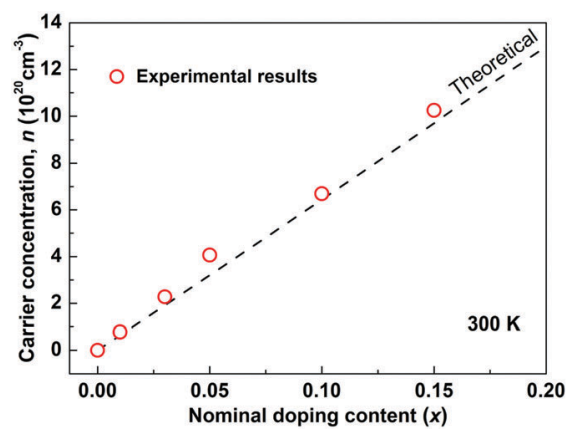

Fig. 2 Carrier concentration as a function of the nominal doping content at room temperature in $\mathrm{Cu}_{3} \mathrm{Sb}_{1-x} \mathrm{Sn}_{x} \mathrm{~S}_{4}$ system. The dashed line is the theoretical model where a full charge transfer is assumed (one hole carrier per dopant).
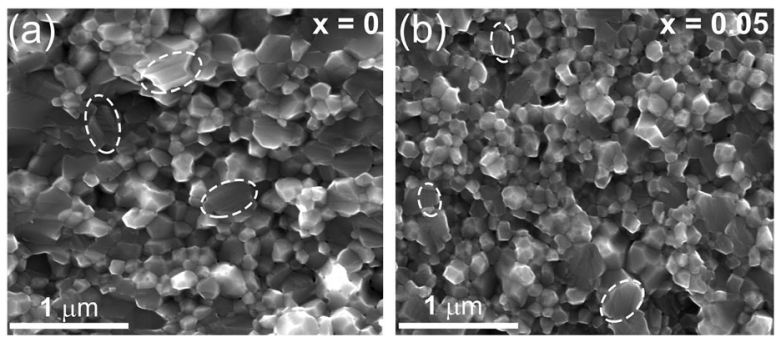

Fig. 3 Representative SEM images of fractured surface for $\mathrm{Cu}_{3} \mathrm{Sb}_{1-x} \mathrm{Sn}_{x} \mathrm{~S}_{4}$, (a) $x=0$, (b) $x=0.05$. Striation-like defects were observed on the transgranular fracture of the grains.

$400 \mathrm{~nm}$, which is much smaller than the reported grain size of 3 to $10 \mu \mathrm{m}$ of $\mathrm{Cu}_{3} \mathrm{SbS}_{4}$ prepared by solid state reaction. ${ }^{10}$ According to previous reports, these nanoscale grains and mesoscale grains in $\mathrm{Cu}_{3} \mathrm{SbS}_{4}$ can effectively scatter phonons with medium- and long-wavelengths. ${ }^{41-43}$ Looking closely at the microstructures, some striation-like defects were observed on the transgranular fractured grains for both undoped and doped samples and these were examined by TEM. Fig. 4a shows a representative low magnification TEM image of $\mathrm{Cu}_{3} \mathrm{SbS}_{4}$, and the striation-like defects can be seen more clearly. These defects were identified as being due to crystal twining from high resolution transmission electron microscopy (HRTEM) images. A series of twin bands with thickness from $\sim 2 \mathrm{~nm}$ to $\sim 16 \mathrm{~nm}$ can be seen in Fig. $4 \mathrm{~b}$. Fig. $4 \mathrm{c}$ shows the typical twin structure of two domains with mirror symmetry and the measured $d$-spacing of domain I and domain II are approximately $0.314 \mathrm{~nm}$, consistent with the (112) planes in the $\mathrm{XRD}$ data of $\mathrm{Cu}_{3} \mathrm{SbS}_{4}$. A corresponding selected area electron diffraction (SAED) pattern is shown in Fig. 4d. Two sets of symmetric electron diffraction spots were observed in the pattern along the $[0-21]$ zone axis, and the twin planes are confirmed as $\{112\}$ planes. Similar twinning defects were also found in the Sn-doped samples (see Fig. S1 in ESI $\dagger$ ). The TEM analysis is consistent with the reported twinning structures in $\mathrm{Cu}_{2} \mathrm{ZnSnS}_{4}$, which has a similar tetragonal zinc blende related structure to $\mathrm{Cu}_{3} \mathrm{SbS}_{4} \cdot{ }^{44,45}$ The twinning structures reported in InSb and $\mathrm{ZnS}$ with cubic zinc blende structure, are on the $\{111\}$ close-packed planes. ${ }^{46,47}$

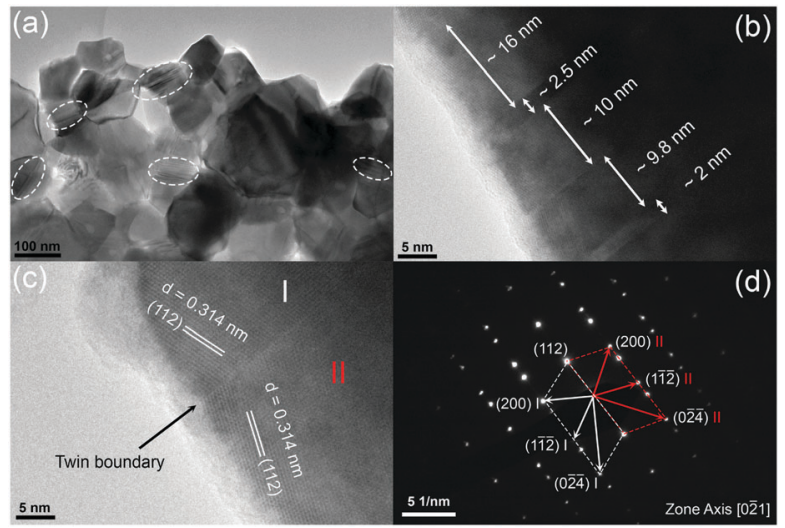

Fig. 4 Representative TEM images for $\mathrm{Cu}_{3} \mathrm{SbS}_{4}$, (a) low magnification image, (b and c) HRTEM images with twinning superlattice, and (d) corresponding SAED pattern. 
These results are also consistent as the $\{112\}$ planes in $\mathrm{Cu}_{3} \mathrm{SbS}_{4}$ are equivalent to the $\{111\}$ planes in the cubic lattice. The above microstructural features of $\mathrm{Cu}_{3} \mathrm{SbS}_{4}$ were not noticed in our previous work ${ }^{9}$ and have not been reported by others. There is, in fact, very limited research on the effects of nano-twinning in TE materials. The nano-twinning defects in $\mathrm{Cu}_{3} \mathrm{SbS}_{4}$ are most likely to be deformation twins. As the samples were prepared by MA + SPS, the high energy collisions in MA and the fast sintering with high pressure in SPS could all contribute to the formation of these defects. When considering the impact of nano-twinning on the transport properties, the twin boundary can be regarded as a coherent boundary with atomic structure close to the perfect crystal. ${ }^{42,48,49}$ Compared to an incoherent grain boundary, a coherent twin boundary is less effective in scattering phonons, but has far less effect on carrier transport. ${ }^{50-52}$ It is rationalized that a high density of twin boundaries can introduce noticeable additional scattering of phonons with nearly unaffected electrical transport.

The temperature dependences of the electrical resistivity $(\rho)$ and Seebeck coefficient $(\alpha)$ are shown in Fig. 5a and b, respectively. The undoped $\mathrm{Cu}_{3} \mathrm{SbS}_{4}$ sample has a carrier concentration $(n)$ of $3.2 \times 10^{17} \mathrm{~cm}^{-3}$ at room temperature and showed semiconducting behavior with a large electrical resistivity and Seebeck coefficient, both of which decreased with increasing temperature. The electrical resistivities of Sn-doped samples were significantly reduced due to the increased carrier concentration (Fig. 2) and consequently, their Seebeck coefficients decreased. All of the Sn-doped samples exhibited metal-like behavior, and the electrical resistivity and Seebeck coefficient increased with increasing temperature. This is opposite to the trend seen in the undoped sample. Fig. 5c shows the carrier concentration dependence of the Seebeck coefficient at $300 \mathrm{~K}$, including previously reported data for $\mathrm{Cu}_{3} \mathrm{SbS}_{4} \cdot^{9-11}$
A single parabolic band (SPB) model was used to evaluate the transport properties (see ESI $\dagger$ for SPB calculations) ${ }^{53}$ and the dashed line represents calculated results with a density of state (DOS) effective mass $\left(m^{*}\right)$ of $3.0 m_{\mathrm{e}}$. The results show very good consistency, indicating a large effective mass in $\mathrm{Cu}_{3} \mathrm{SbS}_{4}$ despite the different doping levels. The power factor was greatly enhanced with Sn-doping, and a maximum power factor of $1.35 \mathrm{~mW} \mathrm{~K}^{-2} \mathrm{~m}^{-1}$ was obtained at $573 \mathrm{~K}$ for $x=0.1$ sample. To identify the optimum carrier concentration $\left(n_{\text {opt. }}\right)$, the carrier concentration dependence of the power factor at $573 \mathrm{~K}$ is shown in Fig. 5d, assuming the carrier concentration is constant with increasing temperature. The experimental results are very close to the SPB predictions with effective mass of $3.0 \mathrm{~m}_{\mathrm{e}}$ to $3.6 m_{\mathrm{e}}$, indicating an optimum carrier concentration around $5 \times 10^{20} \mathrm{~cm}^{-3}$. The power factor reported by Goto et al. (ref. 11) is much lower, which was probably caused by the second phase of $\mathrm{SnO}_{2}$ in their material. ${ }^{11}$ The optimum carrier concentration corresponding to the maximum power factor and the effective mass $m^{*}$ of Sn-doped $\mathrm{Cu}_{3} \mathrm{SbS}_{4}$ identified in this work match well with the empirical relationship of $n_{\text {opt. }} \propto m^{*} .^{54,55}$ Fig. 6 a shows the theoretical values of the Seebeck coefficient obtained from the HSE06-DFT bands of pristine $\mathrm{Cu}_{3} \mathrm{SbS}_{4}$, which are in very good agreement with the experimental data and consistent with our previous DFT $+U$ calculations. ${ }^{9}$ It is important to point out that, as shown in Fig. 6b, the simple model for doping based on the electronic bands of $\mathrm{Cu}_{3} \mathrm{SbS}_{4}$ and a shift of the chemical potential gives results in good agreement with the ones obtained using the built model of $\mathrm{Cu}_{3} \mathrm{Sb}_{0.875} \mathrm{Sn}_{0.125} \mathrm{~S}_{4}$, thus providing a clear validation of the theoretical approach. The choice of the HSE06 hybrid functional offers a way to bypass the need to use the adjustable $U$ parameter of $\mathrm{DFT}+U$, while retaining excellent structural and electronic properties. ${ }^{32,56-58}$ To show this, the accuracy of the HSE06 results were ultimately
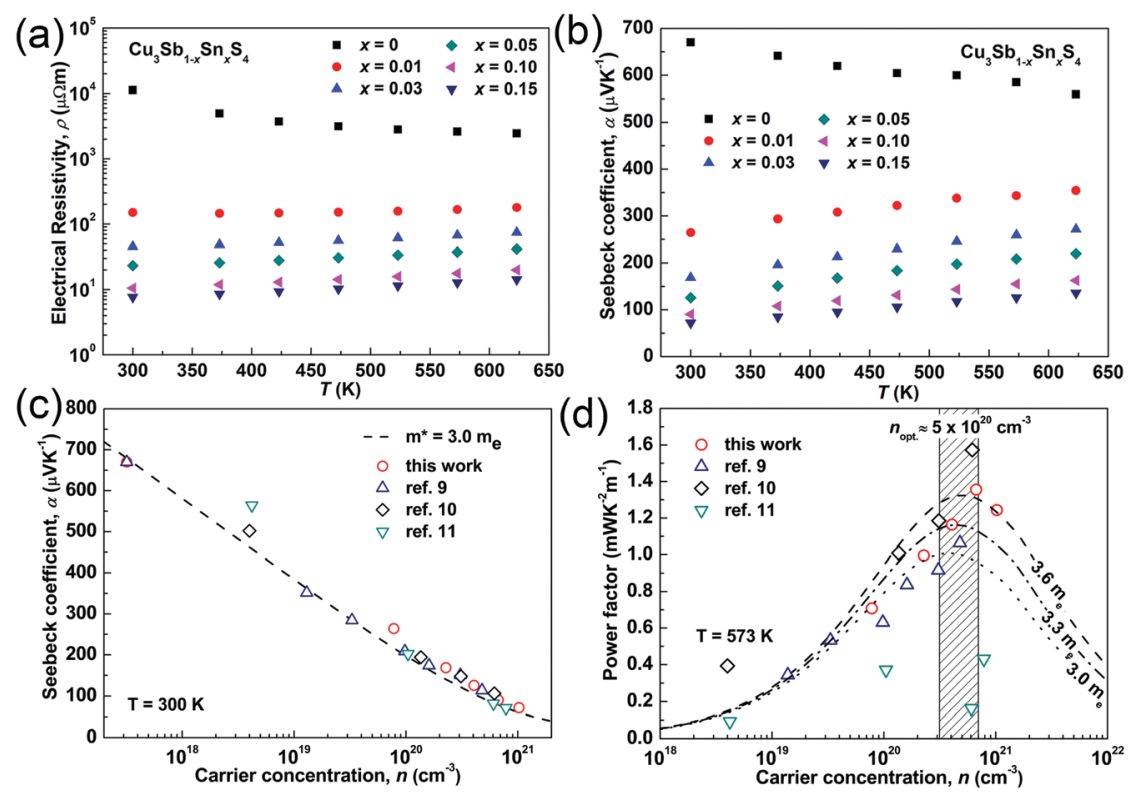

Fig. 5 (a) Electrical resistivity and (b) Seebeck coefficient as functions of temperature; (c) Seebeck coefficient and (d) power factor as functions of carrier concentration in $\mathrm{Cu}_{3} \mathrm{Sb}_{1-x} \mathrm{Sn}_{x} \mathrm{~S}_{4}$ system. 
(a)

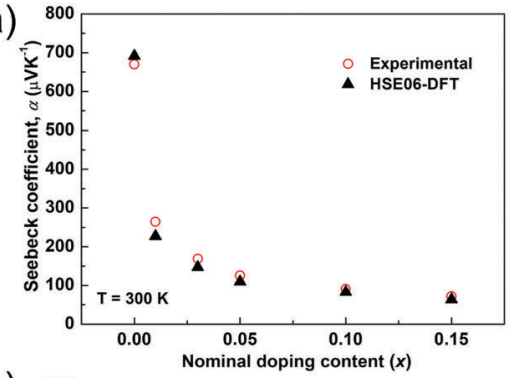

(b)

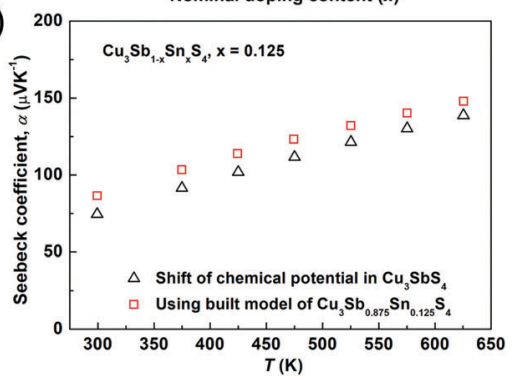

Fig. 6 (a) Comparison of experimental and theoretical Seebeck coefficients in the $\mathrm{Cu}_{3} \mathrm{Sb}_{1-x} \mathrm{Sn}_{x} \mathrm{~S}_{4}$ system at $300 \mathrm{~K}$. The doping was simulated via a shift of chemical potential based on the $\mathrm{Cu}_{3} \mathrm{SbS}_{4}$ structure. (b) The calculated Seebeck coefficient of $\mathrm{Cu}_{3} \mathrm{Sb}_{1-x} \mathrm{Sn}_{x} \mathrm{~S}_{4}(x=0.125)$ as a function of temperature. Open triangles represent the results based on the $\mathrm{Cu}_{3} \mathrm{SbS}_{4}$ structure with a shift of the chemical potential and open squares represent the results based on the built model of $\mathrm{Cu}_{3} \mathrm{Sb}_{0.875} \mathrm{Sn}_{0.125} \mathrm{~S}_{4}$.

assessed against state-of-the-art QSGW calculations. Fig. 7 shows the electronic band structures of $\mathrm{Cu}_{3} \mathrm{SbS}_{4}$ calculated by both methods. The top of the valence band (the part that determines the transport properties of the p-doped compounds) is very similar in the two calculations: for instance, the density of states effective mass, $m^{*}$, (computed neglecting spin-orbit coupling) is $2.2 m_{\mathrm{e}}$ using HSE06 and $2.4 m_{\mathrm{e}}$ using QSGW, confirming the $\mathrm{DFT}+U$ prediction of a high $m^{*}$ to explain the favorably large Seebeck coefficient in this compound. ${ }^{9}$ We notice that the spin-orbit coupling leads to a relatively small splitting of the degenerate bands at the valence band maximum (the splitting is $0.06 \mathrm{eV}$ in QSGW and $0.03 \mathrm{eV}$ in DFT). For completeness, it is important to point out that the two band structures also present

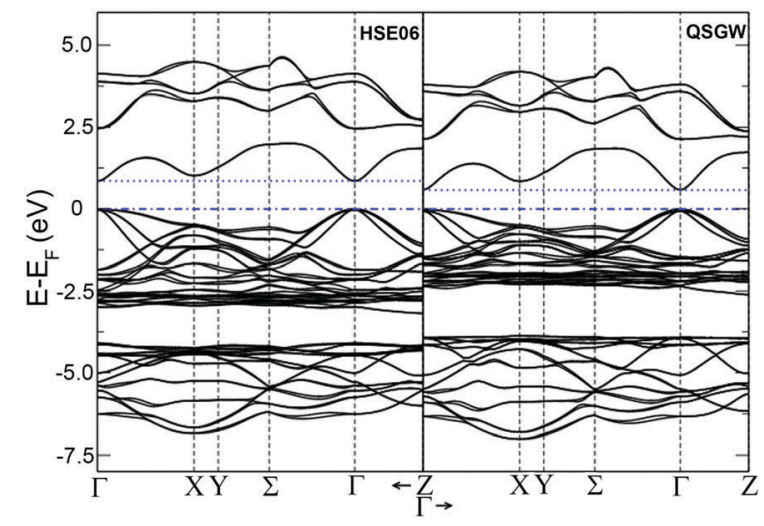

Fig. 7 Band structure for $\mathrm{Cu}_{3} \mathrm{SbS}_{4}$ for spin-orbit coupling calculation with hybrid-DFT (HSE06, left panel) and QSGW (right panel). All the energies in eV referred to the Fermi energy. noticeable differences. First, the valence band from the HSE06 calculations appears broader in energy than that from QSGW, because the fraction of non-local exchange in HSE06 generally tends to stretch the density of states over a wider range of energies. ${ }^{59}$ Secondly, and more importantly, the predicted band gap from HSE06 $\left(E_{\mathrm{g}}=0.85 \mathrm{eV}\right)$ is larger than that from QSGW band gap $\left(E_{\mathrm{g}}=0.58 \mathrm{eV}\right)$. The latter is closer to the experimental value of $\sim 0.47 \mathrm{eV} .{ }^{24}$ These differences are, however, not expected to affect our prediction for the Seebeck coefficient of $\mathrm{Cu}_{3} \mathrm{SbS}_{4}$ compound in the region of different doping levels.

Fig. $8 \mathrm{a}$ and $\mathrm{b}$ show the temperature dependence of the thermal conductivities $(\kappa)$ and lattice thermal conductivities $\left(\kappa_{\mathrm{L}}\right)$. For all of the samples, the thermal conductivity decreased with increasing temperature and there was no sign of bipolar effects over the investigated temperature range. With increasing $\mathrm{Sn}$ doping, the thermal conductivity increased due to the increased electronic contribution. The lattice thermal conductivity was calculated by subtracting the electronic contribution from the thermal conductivity. The Lorenz numbers were calculated based on the SPB model (see Fig. S2 in ESI $\dagger$ ). As seen in Fig. 8b, all of the samples in the present work have similar lattice thermal conductivity and follow a $T^{-1}$ trend, suggesting that thermal conduction is limited by Umklapp scattering and Sn-doping has little impact on lattice thermal conductivity. ${ }^{60}$ Compared to the reported results for $\mathrm{Cu}_{3} \mathrm{SbS}_{4},{ }^{10,11,26}$ the sample prepared in this work shows a very low
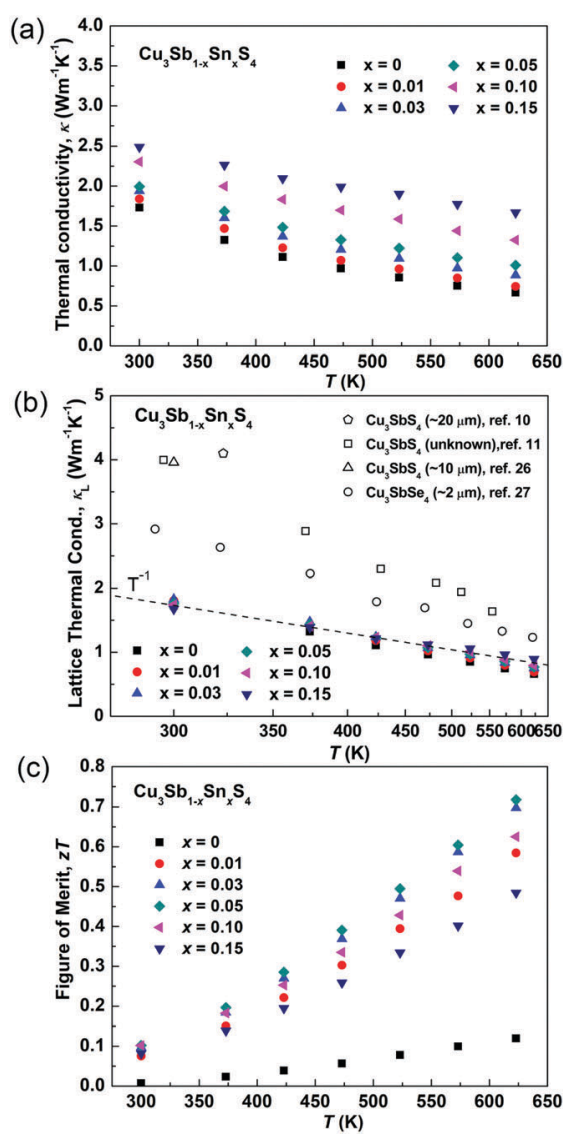

Fig. 8 (a) Thermal conductivity, (b) lattice thermal conductivity and (c) $z T$ values of as functions of temperature in $\mathrm{Cu}_{3} \mathrm{Sb}_{1-x} \mathrm{Sn}_{x} \mathrm{~S}_{4}$ system. 
lattice thermal conductivity, which is more than two times lower at room temperature. It is even lower than that reported in its analogue $\mathrm{Cu}_{3} \mathrm{SbSe}_{4} \cdot{ }^{27}$ This greatly reduced lattice thermal conductivity can be attributed to the unique microstructures of the materials produced in the current work. The extra phonon scattering is from the high density of grain boundaries created by the nanoscale and mesoscale grains, while the previously reported samples had microscale grains. The grain boundaries can effectively scatter medium- and long-wavelength phonons. The nano-twinning boundaries could also contribute to the low lattice thermal conductivity in $\mathrm{Cu}_{3} \mathrm{SbS}_{4}$, although it was reported to be less effective at phonon scattering than grain boundaries in several high thermal conductivity systems, such as diamond and $\mathrm{Si}^{50,51}$ As all the samples in the present study have similar microstructures, we are not able to clarify the individual effect from grain boundaries and nano-twinning boundaries in this work. Nevertheless, it is worth paying more attention to the microstructures of compounds with similar structures. Fig. 8c shows the temperature dependence of the figure-of-merit, $z T$. The $z T$ values exhibit a linearly increasing trend with increasing temperature for all of the samples. A maximum $z T$ value of 0.72 was obtained at $623 \mathrm{~K}$ in the $x=0.05$ sample. It is the highest $z T$ value ever reported for a $\mathrm{Cu}_{3} \mathrm{SbS}_{4}$ based compound, which is comparable or even higher than the reported value of the Se analogue $\mathrm{Cu}_{3} \mathrm{SbSe}_{4} \cdot{ }^{27,61-65}$

\section{Conclusions}

Sn-Doping in $\mathrm{Cu}_{3} \mathrm{SbS}_{4}$ leads to a solid solution of general formula $\mathrm{Cu}_{3} \mathrm{Sb}_{1-x} \mathrm{Sn}_{x} \mathrm{~S}_{4}$. High density samples were prepared by a combination of mechanical alloying and spark plasma sintering, yielding high purity, dense materials with nanoscale and mesoscale grains ( $50 \mathrm{~nm}$ to $400 \mathrm{~nm}$ ). Sn was found to have an exceptionally high solubility and work effectively as a p-type dopant in $\mathrm{Cu}_{3} \mathrm{SbS}_{4}$. The high $x$-value compositions are the subject of further work. Interesting twinning defects on $\{112\}$ planes with nanoscale separation were found in $\mathrm{Cu}_{3} \mathrm{SbS}_{4}$ samples, which have not previously been reported. The electronic structure of this compound was carefully investigated using both HSE06-DFT and QSGW, and the calculated Seebeck coefficient was in very good agreement with the experimental results. A large effective mass $\left(3.0 m_{\mathrm{e}}\right)$ deriving from degenerate bands was found in $\mathrm{Cu}_{3} \mathrm{SbS}_{4}$, which is favorable for high thermoelectric performance. All of the samples in the present work exhibited very low lattice thermal conductivities, due mainly to the high density of grain boundaries. Considering the low lattice thermal conductivity of the samples, the nano-twinning defects are likely to impact on phonon transport. A maximum $z T$ value of 0.72 at $623 \mathrm{~K}$ was achieved in the $x=0.05$ sample with a carrier concentration of $\sim 4.1 \times 10^{20} \mathrm{~cm}^{-3}$. This is the highest $z T$ value reported for $\mathrm{Cu}_{3} \mathrm{SbS}_{4}$ system. Although the effects of twining boundaries on the electron and phonon transport is still not clear, more attention should be paid to nano-twinning in $\mathrm{Cu}_{3} \mathrm{SbS}_{4}$ and related compounds, as well as other thermoelectric materials.

\section{Conflicts of interest}

There are no conflicts to declare.

\section{Acknowledgements}

This work was supported by the Engineering and Physical Sciences Research Council (EPSRC) (Grant No. EP/N0227261/1 and EP/N02396X/1). N. B. and C. W. acknowledge the ARCHER UK National Supercomputing Service and the UK Materials and Molecular Modelling Hub for computational resources, which are partially funded by EPSRC (EP/P020194/1). N. B. also acknowledges the Cirrus UK National Tier-2 HPC Service at EPCC. N. B. and S. L. thank Mark van Schilfgaarde for helping with the QSGW calculations. C. W. and N. B. acknowledge Dr Glenn Jones (Johnson \& Matthey, Sonning) for support and helpful discussions. C. W. gratefully acknowledges the support of NVIDIA Corporation with the donation of the Tesla K40 GPUs used in this research. Dr R. M. Wilson (QMUL) is thanked for his help in collecting XRD data.

\section{Notes and references}

1 C. Forman, I. K. Muritala, R. Pardemann and B. Meyer, Renewable Sustainable Energy Rev., 2016, 57, 1568-1579.

2 D. M. Rowe, CRC Handbook of Thermoelectrics, CRC Press, 1995.

3 G. S. Nolas, J. Sharp and H. J. Goldsmid, Thermoelectrics, 2001.

4 W. Liu, J. Hu, S. Zhang, M. Deng, C.-G. Han and Y. Liu, Mater. Today Phys., 2017, 1, 50-60.

5 M. Takao, Small, 2017, 13, 1702013.

6 S. Koichiro, T. Kojiro, A. Tomoki and K. Mikio, Appl. Phys. Express, 2012, 5, 051201.

7 X. Lu, D. T. Morelli, Y. Xia, F. Zhou, V. Ozolins, H. Chi, X. Y. Zhou and C. Uher, Adv. Energy Mater., 2013, 3, 342-348.

8 R. Chetty, A. Bali and R. C. Mallik, J. Mater. Chem. C, 2015, 3, 12364-12378.

9 K. Chen, B. Du, N. Bonini, C. Weber, H. Yan and M. J. Reece, J. Phys. Chem. C, 2016, 120, 27135-27140.

10 A. Suzumura, M. Watanabe, N. Nagasako and R. Asahi, J. Electron. Mater., 2014, 43, 2356-2361.

11 Y. Goto, Y. Sakai, Y. Kamihara and M. Matoba, J. Phys. Soc. Jpn., 2015, 84, 044706.

12 V. Kumar Gudelli, V. Kanchana, G. Vaitheeswaran, A. Svane and N. E. Christensen, J. Appl. Phys., 2013, 114, 223707.

13 B. Du, R. Zhang, K. Chen, A. Mahajan and M. J. Reece, J. Mater. Chem. A, 2017, 5, 3249-3259.

14 A. U. Khan, R. A. R. A. Orabi, A. Pakdel, J.-B. Vaney, B. Fontaine, R. Gautier, J.-F. Halet, S. Mitani and T. Mori, Chem. Mater., 2017, 29, 2988-2996.

15 Y. W. Shen, C. Li, R. Huang, R. M. Tian, Y. Ye, L. Pan, K. Koumoto, R. Z. Zhang, C. L. Wan and Y. F. Wang, Sci. Rep., 2016, 6, 32501.

16 H. Zhao, X. Xu, C. Li, R. Tian, R. Zhang, R. Huang, Y. Lyu, D. Li, X. Hu, L. Pan and Y. Wang, J. Mater. Chem. A, 2017, 5, 23267-23275. 
17 Y. Yang, P. Ying, J. Wang, X. Liu, Z. Du, Y. Chao and J. Cui, J. Mater. Chem. A, 2017, 5, 18808-18815.

18 N. Tsujii and T. Mori, Appl. Phys. Express, 2013, 6, 043001.

19 D. Berthebaud, O. I. Lebedev and A. Maignan, J. Materiomics, 2015, 1, 68-74.

20 R. Ang, A. U. Khan, N. Tsujii, K. Takai, R. Nakamura and T. Mori, Angew. Chem., Int. Ed., 2015, 54, 12909-12913.

21 H. Takaki, K. Kobayashi, M. Shimono, N. Kobayashi, K. Hirose, N. Tsujii and T. Mori, Mater. Today Phys., 2017, 3, 85-92.

22 G. Guelou, A. V. Powell and P. Vaqueiro, J. Mater. Chem. C, 2015, 3, 10624-10629.

23 R. Zhang, K. Chen, B. Du and M. J. Reece, J. Mater. Chem. A, 2017, 5, 5013-5019.

24 K. I. Amirkhanov, G. G. Gadzhiev and Y. B. Magomedov, High Temp., 1978, 16, 1050-1052.

25 Y. Pei, X. Shi, A. LaLonde, H. Wang, L. Chen and G. J. Snyder, Nature, 2011, 473, 66-69.

26 E. J. Skoug, J. D. Cain, D. T. Morelli, M. Kirkham, P. Majsztrik and E. Lara-Curzio, J. Appl. Phys., 2011, 110, 023501.

27 T. Wei, H. Wang, Z. M. Gibbs, C. Wu, G. J. Snyder and J. Li, J. Mater. Chem. A, 2014, 2, 13527-13533.

28 G. Kresse and D. Joubert, Phys. Rev. B: Condens. Matter Mater. Phys., 1999, 59, 1758-1775.

29 G. Kresse and J. Hafner, Phys. Rev. B: Condens. Matter Mater. Phys., 1994, 49, 14251-14269.

30 J. Heyd, G. E. Scuseria and M. Ernzerhof, J. Chem. Phys., 2006, 124, 219906.

31 J. Heyd, G. E. Scuseria and M. Ernzerhof, J. Chem. Phys., 2003, 118, 8207-8215.

32 J. Paier, M. Marsman, K. Hummer, G. Kresse, I. C. Gerber and J. G. Ángyán, J. Chem. Phys., 2006, 124, 154709.

33 J. Paier, M. Marsman, K. Hummer, G. Kresse, I. C. Gerber and J. G. Ángyán, J. Chem. Phys., 2006, 125, 249901.

34 G. Pizzi, D. Volja, B. Kozinsky, M. Fornari and N. Marzari, Comput. Phys. Commun., 2014, 185, 422-429.

35 T. Kotani, M. van Schilfgaarde and S. V. Faleev, Phys. Rev. B: Condens. Matter Mater. Phys., 2007, 76, 165106.

36 M. van Schilfgaarde, T. Kotani and S. Faleev, Phys. Rev. Lett., 2006, 96, 226402.

37 M. van Schilfgaarde, T. Kotani and S. V. Faleev, Phys. Rev. B: Condens. Matter Mater. Phys., 2006, 74, 245125.

38 R. Shannon, Acta Crystallogr., Sect. A: Cryst. Phys., Diffr., Theor. Gen. Crystallogr., 1976, 32, 751-767.

39 J. Garin and E. Parthé, Acta Crystallogr., Sect. B: Struct. Crystallogr. Cryst. Chem., 1972, 28, 3672-3674.

40 C. D. Paola, K. Chen, I. Abrahams, N. Bonini, C. Weber, $\mathrm{H}$. Yan and M. Reece, in preparation.
41 K. Biswas, J. He, I. D. Blum, C. I. Wu, T. P. Hogan, D. N. Seidman, V. P. Dravid and M. G. Kanatzidis, Nature, 2012, 489, 414-418.

42 G. J. Tan, L. D. Zhao and M. G. Kanatzidis, Chem. Rev., 2016, 116, 12123-12149.

43 D. M. Rowe, V. S. Shukla and N. Savvides, Nature, 1981, 290, 765.

44 B. Shin, Y. Zhu, T. Gershon, N. A. Bojarczuk and S. Guha, Thin Solid Films, 2014, 556, 9-12.

45 N. Kattan, B. Hou, D. J. Fermín and D. Cherns, Appl. Mater. Today, 2015, 1, 52-59.

46 Y. Qian and Q. Yang, Nano Lett., 2017, 17, 7183-7190.

47 H. B. Ahn and J. Y. Lee, Mater. Lett., 2013, 106, 308-312.

48 R. Aghababaei, G. Anciaux and J.-F. Molinari, Appl. Phys. Lett., 2014, 105, 194102.

49 J. He, M. G. Kanatzidis and V. P. Dravid, Mater. Today, 2013, 16, 166-176.

50 H. Dong, J. Xiao, R. Melnik and B. Wen, Sci. Rep., 2016, 6, 19575.

51 H. F. Zhan, Y. Y. Zhang, J. M. Bell and Y. T. Gu, J. Phys. D: Appl. Phys., 2014, 47, 015303.

52 L. Lu, Y. Shen, X. Chen, L. Qian and K. Lu, Science, 2004, 304, 422-426.

53 D. M. Rowe, Thermoelectrics and its energy harvesting, CRC Press, Boca Raton, FL, 2012.

54 C. Fu, S. Bai, Y. Liu, Y. Tang, L. Chen, X. Zhao and T. Zhu, Nat. Commun., 2015, 6, 8144.

55 C. Fu, T. Zhu, Y. Liu, H. Xie and X. Zhao, Energy Environ. Sci., 2015, 8, 216-220.

56 J. Paier, R. Asahi, A. Nagoya and G. Kresse, Phys. Rev. B: Condens. Matter Mater. Phys., 2009, 79, 115126.

57 F. Fuchs, J. Furthmüller, F. Bechstedt, M. Shishkin and G. Kresse, Phys. Rev. B: Condens. Matter Mater. Phys., 2007, 76, 115109.

58 M. Jain, J. R. Chelikowsky and S. G. Louie, Phys. Rev. Lett., 2011, 107, 216806.

59 J. E. Northrup, M. S. Hybertsen and S. G. Louie, Phys. Rev. Lett., 1987, 59, 819-822.

60 E. S. Toberer, A. Zevalkink and G. J. Snyder, J. Mater. Chem., 2011, 21, 15843.

61 X. Y. Li, D. Li, H. X. Xin, J. Zhang, C. J. Song and X. Y. Qin, J. Alloys Compd., 2013, 561, 105-108.

62 C. Yang, F. Huang, L. Wu and K. Xu, J. Phys. D: Appl. Phys., 2011, 44, 295404.

63 E. J. Skoug, J. D. Cain, P. Majsztrik, M. Kirkham, E. Lara-Curzio and D. T. Morelli, Sci. Adv. Mater., 2011, 3, 602-606.

64 D. Li, R. Li, X. Qin, C. Song, H. Xin, L. Wang, J. Zhang, G. Guo, T. Zou, Y. Liu and X. Zhu, Dalton Trans., 2014, 43, 1888-1896.

65 D. Chen, Y. Zhao, Y. Chen, T. Lu, Y. Wang, J. Zhou and Z. Liang, Adv. Electron. Mater., 2016, 2, 1500473. 